\title{
ASPECTS ACTUELS DE LA RECHERCHE FRANÇAISE EN HISTOIRE DU MOYEN ÂGE.
}

\author{
JEAN GLENISSON \\ Ecole Pratique des Hautes Etudes. 6ème Section, Paris. \\ Ancien professeur à l'Université de São Paulo.
}

Qui croirait que l'histoire elle-même a sa place dans la planification "à la française"? Tous les cinq ans, la préparation du Plan de développement qui est censé encadrer et régir l'activité nationale donne l'occasion aux historiens français de s'interroger à la fois sur l'évolution de la discipline qu'ils cultivent dans les cinq années écoulées, et sur l'aide que l'Etat pourrait apporter au développement de leurs études.

Mil neuf cent soixante quatorze a été l'une de ces années de réflexion et de proposition. Sous la signature abusive d'un ancien professeur à l'Université de São Paulo, les pages qui suivent reprennent les informations et les observations à lui, fournies par de nombreux médiévistes, ses amis et ses collègues (1). L'hospitalité offerte par la Revista de História permet de soumettre ces notes à des historiens qui, peut-être, n'auraient pas eu l'occasion d'en rechercher la version "officielle", dans un rapport administratif.

(1). - Il s'agit plus particulièrement de Philippe Contamine, Robert Fossier, Bernard Guenée, Bernard Guillemain, Jacques Monfrin, Jean Vezin à qui ont été empruntés non seulement des observations précises, mais aussi des passages entiers des notes qu'ils m'ont remises à l'occasion des travaux préliminaires du rapport sur l'histoire du Moyen Age, rédigé pour le compte du Centre Nationale de Recherche Scientifique, à l'occasion de la préparation du "VIIème Plan". 
Les "scientifiques" qui tiennent le haut du pavé dans la recherche contemporaine s'étonnent souvent de la lenteur des changements dans les sciences de l'homme. Les historiens savent bien, en revanche, que dans leur domaine, les progrès reposent en fin de compte sur la découverte, l'analyse et la publication des sources nouvelles, dont l'exploitation est longue et difficile, autant que sur le renouvellement des mentalités et la relève des générations.

Rien d'étonnant, dès lors, que l'histoire du Moyen Âge soit marquée, en France, par des tendances qui commençèrent à s'affirmer avec insistance dès les années 1930, pour ne triompher sans conteste qu'au lendemain de la seconde guerre mondiale.

L'oeuvre et l'influence de Marc Bloch et de ses disciples font que les liens, aujourd'hui presque indissolubles, qui unissent l'économique et le social demeurent le trait dominant de l'histoire du Moyen Âge, telle qu'elle est conçue chez nous depuis plusieurs décennies. Il est vrai aussi que l'histoire économique aurait quelque mal à se dissocier vraiment de l'histoire sociale, lorsqu'il faut traiter d'une époque dont les sources subsistantes sont à la fois trop incomplètes et trop incertaines pour qu'il soit possible d'avoir régulièrement et constamment recours aux méthodes statistiques et économiques.

Dans le champ plus spécifiquement économique, il convient cependant de noter, en France, les progrès - encore insuffisants des études consacrées aux phénomènes monétaires, l'attention nouvelle portée aux mécanismes économiques, enfin et surtout, la remise en cause de la notion de "crise" qui a dominé la réflexion et inspiré les travaux de plusieurs générations de médiévistes, ainsi voués inexorablement à l'étude de la période de la longue dépression qui s'étend des années 1280 au dernier quart du XVe siècle (2). Dans le domaine plus proprement social, l'intérêt est resté vif pour la génèse et les transformations de la seigneurie, l'idée d'un processus généralisé de réduction de la réserve étant de plus en plus battue en brèche; la condition paysanne est scrutée avec une attention particulière. Mais il faut surtout noter l'apparition d'un courant de recherche consacré

(2). - L'intérêt pour les questions monétaires est notable avec la réédition du livre de M. LOMBARD, Monnaie et histoire d'Alexandre à Mahomet, Paris - La Haye 1971 et avec la synthèse de E. FOURNIAL, Histoirt! monétaire de l'Occident médiéval, collection Fac., Paris 1970. Crises locales mises en évidence avec l'étude de R. FEDOU, Le cycle médiéval des révoltes lyonnaises, parue en 1973 à Lyon. M. MOLIAT et Ph. WOLFF ont présenté une esquisse européenne des mouvements populaires avec leur ouvrage commun Ongles bleus, Jacques et Ciompi: Les Révolutions populaires en Europe aux XIVe et XVe, Paris, 1970. Dans la collection SUP., l'Historien, G. FOURQUIN présente Les soulèvements populaires au Moyen-Age, Paris, 1972: 
aux groupes marginaux de la société médiévale (3). De notables progrès ont été faits dans la connaissance de l'implantation et de la condition des juifs en France, grâce au repérage systématique des sources de toute nature. Le prolétariat urbain, les pauvres, les mendiants, les criminels ont suscité de nombreux travaux, par référence implicite, sans doute, à la situation actuelle du monde. La réflexion sur le présent se projette aussi dans le passé, avec la prolifération récente des études sur les révoltes médiévales, leurs acteurs et leurs mécanismes, non tant aux XIVe et XVe siècles bien connus, qu'au cours de la période "révolutionnaire" du XIe siècle, ainsi que sur les classes d'âge et le rôle des jeunes dans l'Occident médiéval. Peu à peu s'édifie une sociologie rétrospective du Moyen Âge, qui use de la prosopographie, qui étudie les "ordres", les catégories sociales, les groupes, dont elle s'efforce de déceler les motivations collectives, de mesurer le poids dans l'ensemble social, d'analyser le jeu dans le mécanisme politique, de démêler les relations internes et de découvrir les moyens d'action .

Dans le vaste domaine d'une histoire qui lie l'économique et le social, l'un des traits majeurs est le considérable accroissement d'intérêt qui s'est manifesté, au cours des cinq dernières années, pour les études d'histoire agraire (4). Le XIXe siècle, d'Augustin Thierry à

(3). - Les groupes marginaux ont été étudiés soit par des ouvrages de synthèse ou des publications de documents tels que Confessions et jugements de criminels au Parlement de Paris, 1319-1350, Paris, 1971. B. GEREMEK s'est attaché à dégager Les milieux marginaux à Paris au Moyen Age, XIVe$X V e$, Wroclaw 1971. Y. DOSSAT publie quelques documents inédits sur les Juifs à Toulouse à la fin du XIIIe et au début du XIVe dans le Bulletin philologique et historique, 1969. J. SHATZMILLER nous livre ses Recherches sur la communauté juive de Manosque au Moyen-Age, Paris, La Haye, 1973.

(4). - La transformation de la seigneurie a été mise en évidence dans le tome II de R. BOUTRUCHE, Seigneurie et féodalité, L'apogée XIe-XIIIe, Paris, 1970 ainsi que dans l'ouvrage de A. LEGUAI, De la seigneurie d̀ l'état, le Bourbonnais pendant la guerre de cent ans, Moulins, 1969. La ruralisation de l'histoire est effective lorsque l'on dresse la liste des thèses récentes consacrées à ces problèmes. Citons $\mathrm{M}$. T . LORCIN, Les campagnes dans la région lyonnaise aux XIVe et XVe. Lyon, 1974, G. DEVAILLY, Le Berri du Xème au milieu du XIIIème, Etude politique, religieuse, sociale, économique, Paris, 1973. Les thèses de G. BOIS et de H. BUR qui ont été brillamment soutenues au printemps 1974 seront publiées prochainement: Recherches sur l'économie rurale et la démographie du début XIVe au milieu du XVIe en Normandie orientale. et La formation du comté de Champagne du Xe au XIIe. Etude régionale et rurale avec l'importante thèse de $\mathrm{R}$. FOSSIER: La terre et les hommes en Picardie jusqu'à la fin du XIIIe, Paris, 1968.

G. FOURQUIN a écrit trois ouvrages de synthèse présentant les problèmes ruraux et leur implication dans l'histoire de la féodalité. Ce sont: Seigneurie et Féodalité dans la collection SUP., L'Historien, Paris, 1970, Le Paysan d'Occident au Moyen-Age, Paris, 1972, enfin dans la collection U, série Moyen-Age, Histoire économique de l'Occident médiéval, Paris, 1971. 
Henri Pirenne, avait eu "son" Moyen-Age urbain et bourgeois; la "ruralisation" de l'histoire est l'une des caractéristiques de l'historiographie contemporaine dont il faudrait sans doute chercher les raisons dans une "idéologie" qu'il ne nous appartient pas ici d'analyser. Cet essor des études rurales se manifeste par l'activité des recherches théoriques - compréhension plus nette des liens entre l'histoire des sociétés rurales et celle des techniques; intérêt porté aux types de production (place du mercantilisme, traces de précapitalisme à la campagne) - et méthodologiques: la plupart des nouveautés introduites dans la réflexion historique, les emprunts à la sociologie, à l'anthropologie, l'utilisation des moyens scientifiques de datation, les recherches sur les variations végétales ou pédologiques etc... ont beaucoup plus servi à l'histoire des campagnes qu'à celle des villes. Dans un panorama d'ensemble très favorable, la recherche française occupe une bonne place. Ses principales faiblesses consistent dans un certain manque d'intérêt pour la période du grand essor (Xe-XIIIe siècle.) et dans la préférence manifeste qu'elle accorde aux éléments sociaux dans le tableau de la vie agraire.

Eclipsée dans l'ensemble par l'histoire rurale, l'histoire urbaine (5) n'a cependant pas perdu sa vigueur dans divers pays d'Europe: Allemagne, Belgique, Pologne notamment. Elle se concentre sur la période de l'origine de la ville médiévale, pour laquelle les sources sont rares ou peu explicites. Elle est donc, elle aussi, amenée à rechercher le concours d'autres disciplines: archéologie, topographie, numismatique etc... Ainsi l'image de la ville du Moyen-Age apparaîtelle de plus en plus diversifiée. La science française est caractérisée, dans ce secteur, par un retard manifeste. Elle ne se livre guère aux recherches sur le terrain. Elle concentre ses efforts sur le bas MoyenAge, fertile en documents, et sur les aspects sociologiques de la vie urbaine. Un effort est fait, actuellement, pour saisir par le biais de l'implantation des Ordres mendiants dans les villes françaises médiévales, le phénomène urbain dans son ensemble.

(5). - L'Histoire urbaine est illustrée par plusieurs ouvrages, dont les thèses récentes de $R$. FAVREAU et $H$. DUBOIS qui seront publiés prochainement: R. FAVREAU, Poitiers à la fin du Moyen-Age: Les fonctions d'une capitale régionale et $\mathrm{H}$. DUBOIS, Les foires de Châlon-sur-Saône et le commerce dans la vallée de la Saône à la fin du Moyen-Age. J. FAVIER s'est attaché à définiri le rôle de Paris dans son article: Une ville entre deux vocations: la place d'affaires de Paris au XVe, publié dans les Annales en 1973. Vie d'une province et de sa capitale dans l'ouvrage de P. L. MALAUSSENA, La vie en Provence orientale aux XIVe et XVe, un exemple: Grasse à travers les actes notarićs, Paris, 1969. Problèmes urbains et problèmes de construction auxquels S. ROUX s'est consacré dans L'Habitat urbain au Moyen-Age, le quartier de l'Université à Paris, publié aux Annales en 1969 et dans son livre: La construction courante à Paris du milieu XIVe à la fin XVe, Paris 1973. 
Mais c'est à peine si a été abordée l'étude des liens entre ville et campagne, entre fortunes marchandes et fortunes foncières. Presque rien n'a été fait, non plus, pour saisir l'emprise du capital urbain sur le plat pays, ni le rôle des gains ruraux en ville. Alors qu'historiens anglais et allemands se préoccupent des itinéraires et des marchés, la recherche française demeure attachée à l'étude du grand commerce: à cet égard, son apport à la connaissance du commerce des grains, du bois, du vin, du sel, est apprécié. Toutefois, seule une mise en oeuvre systématique des sources comptables permettrait d'atteindre les transactions modestes, celles vers lesquelles l'attention de l'historien est de plus en plus sollicitée.

Ceux qui pourraient croire encore que les historiens avancent méthodiquement dans la recherche s'étonneront seuls que l'histoire démographique (6), soutien évident, fondement indispensable de toute

(6). - Les Annales de la faculté des Lettres et Sciences humaines de Nice ont publié en 1972 trois articles de fond touchant la démographie étudiée à partir d'exemples provinciaux. Ce sont: A. LEGUAI, Démographie médiévale dans le duché de Bourgogne, sources et méthodes; E. BARATIER, Dímographie médiévale dans le Midi máditerranéen, sources et méthodes; $H$. DUBOIS, Histoire démographique de Châlons-sur-Saône de la fin du XIIe au début du XVe. Problèmes de méthode avec l'article de $J$. HEERS: Les Limites des méthodes statistiques pour les recherches de démographie médiévale, qui a paru dans les Annales de démographie historique en 1969.

La collaboration de M. ROUCHE avec des médecins pour la rédaction de son article: La faim à l'époque carolingienne: essai sur quelques types de rations alimentaires, publié à Paris en 1973 dans la Revue historique ouvre la voie à la biologie historique.

L'étude du costume est un révélateur de la vie sociale. En attendant la thèse que $R$. DELORT consacre au costume, nous pouvons consulter l'ouvrage de F. PIPONNIER, Costume et vie sociale: la Cour d'Anjou aux XIVe et $X V e$. Paris, La Haye 1970. J. HEERS s'est attaché à établir les rapports entre La mode et les marchés des draps de laine: Gênes et la montagne d la fin du Moyen-Age, Paris 1971.

L'Histoire fiscale est essentiellement illustrée par les travaux de J. FAVIER, Finances et fiscalité au Moyen-Age, publié à Paris en 1971 et Les contribuables parisiens à la fin de la guerre de cent ans, paru à Genève en 1970. Publication de documents fiscaux: E. BARATIER Enquête sur les droits et revenus de Charles I d'Anjou en Provence 1275-1278, Paris, 1969. (Documents inédits de l'Histoire de France série in $4^{\circ}$ ).

Un ouvrage collectif de synthèse sur Le rôle du sel dans l'histoire a été publié à Paris, en 1968, par M. MOLLAT. R. FAVREAU a étudié le commerce du sel en Poitou à la fin du Moyen-Age dans Le Bulletin philologique et historique du comité des travaux historiques et scientifiques en 1966. 
histoire économique et sociale, n'ait connu qu'un essor très récent. Il est vrai que ce retard relatif explique sans doute qu'on s'efforce aujourd'hui de mettre les bouchées doubles. L'histoire démographique est bien l'un des "chantiers" où les médiévistes contemporains déploient le plus d'activité. Depuis que l'importance de ce secteur de recherche fut révélée au Congrès international des sciences historiques de 1950, les spécialistes se sont regroupés en Associations nationales et internationales. La société qui s'est formée en France a considérablement contribué aux progrès d'une discipline qui a encore pour tâche première de découvrir ses sources et de définir ses méthodes. L'époque médiévale n'est que partiellement "préstatistique": les documents fiscaux, nombreux au XIVe et au XVe siècles, offerts parfois en séries continues, permettent d'user de l'informatique. Dans ce domaine, les chercheurs français. jouent un rôle éminent. en collaboration avec les Américains. Ils sont aussi en bon rang dans l'étude des anciennes "mortalités", considérées du point de vue médical et dans leurs conséquences historiques. La démographie historique se relie par là à une "histoire biologique" qui tend à s'affirmer comme nouvelle discipline, accorde une place éminente à l'étude des maladies, réintroduit non sans prudence la notion de race dans l'histoire, et tire parti des plus récentes découvertes génétiques. La démographie enrichit ainsi l'éventail de ses sources - le corps de l'homme vivant devenant luimême source historique par l'intermédiaire de l'hématologie! - et ajoute encore, aux textes écrits, les témoignages recueillis par l'archéologie de la vie matérielle, lors du repérage et la fouille des villages désertés notamment.

En 1965, dans un autre bilan de l'historiographie française, j'ai déjà eu l'occasion de mettre en évidence le regain d'intérêt qui commençait alors à se manifester pour l'histoire politique — ou, si l'on préfère, pour les études sur l'Etat, les institutions, la guerre. Au vrai, après des décennies de réprobation, il faudrait parler de résurrection, sinon, de réhabilitation.

Le tabou qui, depuis les années 30 , a progressivement voué à la honte et condamné au silence les médiévistes qui s'adonnaient à l'his-

Ch. VILLAIN-GANDOSSI a publié Les comptes du sel (Libro di Ragione et conto di salle) de Fraicesco di Marco Datini pour sa compagnie d'Avignon 1376-1379, Paris, 1969. Très récemment un article de J. HOCQUET s'est attaché à étudier le sel, histoire comparée en Méditerranée dans les Annales, 1974. 
toire politique, naguère triomphante, est un phénomène qui a marqué tout particulièrement l'historiographie française et qu'il conviendra un jour d'étudier historiquement. Il n'a pas été aussi sensible en histoire moderne, moins encore en histoire contemporaine, il a été propre aux milieux universitaires et à l'érudition établie.

Aujourdhui, la politique réapparaît sous la forme de l'histoire de l'Etat (7). Pourquoi ne pas redire ici que le spectacle offert à l'historien par le monde actuel joue son rôle habituel dans cette renaissance?

Il révèle le rôle d'un Etat qui intervient dans tous les domaines de l'activité nationale, soumis aux influences et aux pressions, mais modelant à son tour l'économie et la société après ses décisions d'ordre politique, aussi bien que par les démarches d'une administration partout présente. De divers côtés, on commence à être tenté de transposer cette image dans le passé. On se demande, en tout cas, s'il est légitime de brosser, dans le cadre d'un pays, le tableau de l'activité économique sans tenir compte de l'appareil, impressionnant de tout temps, d'un Etat centralisé; en ignorant aussi bien les répercussions des actes politiques sur les différents secteurs de la vie nationale et l'influence des "groupes de pression", des castes de magistrats et d'officiers sur les décisions du pouvoir.

Particulièrement sensible en France, en raison du discrédit dont l'histoire politique émerge à peine, cette orientation de la recherche est d'ailleurs générale et intéresse avant tout les derniers siècles du Moyen Age. Chaque école nationale y apporte sa contribution relativement faible chez les histoirens allemands qui, de par une tradition séculaire, continuent de se préoccuper plus spécialement des siècles pendant lesquels l'Empire étendait son ombre sur l'Europe; particulièrement notable chez les Américains, sans lesquels l'histoire de l'Etat médiéval, en France et en Italie, serait loin d'être ce qu'elle est. Progressent notamment l'étude des assemblées représentatives que les historiens anglophones poursuivent avec ténacité; l'étude de la propagande politique et celle des révoltes, sur lesquelles l'activité appelle rétrospectivement l'attention. Mais le sujet qui, de loin, est le plus étudié, est celui des finances des Etats d'Occident à la fin du Moyen Age.

(7). - L'Histoire politique a donné lieu à la parution d'un ouvrage de base B. GUENEE, L'Occident aux XIVe et XVe, les Etats, paru dans la collection Nouvelle Clio, Paris, 1971. R. FEDOU a publié L'Etat au Moyen-Âge dans la collection SUP., série l'Historien, Paris, 1971. Dans la collection Dossier Clio, F. AUTRAND vient de faire paraitre Pouvoir et société en France aux XIVe et XVe, Paris, 1974. 
En ce qui concerne plus spécialement l'historiographie française, les recherches sur l'histoire de l'Etat présentent - pour d'évidentes raisons de sources - deux défauts majeurs. Ou bien, elles sont contraintes de considérer les réalités politiques du point de vue du gouvernement central. Ou bien, s'attachant à étudier les réalités d'une province ou d'une faible étendue de territoire, elles généralisent à l'excès leurs résultats, si bien que, dans l'un et l'autre cas, le pays n'apparaît pas dans son épaisseur et dans sa diversité.

Des thèmes majeurs, ont encore insuffisamment attiré l'attention. Par exemple, l'histoire du sentiment national (8), dont il faudrait éclairer la naissance et le développement, si l'on veut faire progresser l'étude des idées, des sentiments, des croyances qui soutiennent l'Etat.

Dans de telles perspectives est-il encore possible de distinguer l'histoire politique de l'histoire institutionnelle, domaine où les juristes l'emportent, en vertu des compartimentages universitaires traditionnels, sur les historiens de formation classique? L'histoire des institutions entend, elle aussi, dépasser l'analyse juridique de la description des structures pour atteindre les réalités sociales, pour connaître le succès ou l'échec des règles et en déterminer les causes. De la description, elle est passée à la réflexion, non pour en tirer d'impossibles lois, mais pour mieux pénétrer la vie des sociétés et les interactions entre règles juridiques et réalités politiques et sociales.

Sans doute l'histoire institutionnelle ne se borne-t-elle pas à l'examen des institutions politiques: l'école française s'est fait une place dans l'étude des institutions chrétiennes d'Occident, et elle entend poursuivre cette oeuvre. Mais historiens et juristes pourraient unir leurs efforts pour analyser la formation de la classe administrative, de la "société politique" françaises, à l'échelon urbain et patronal. Reprenant et amplifiant des travaux qui ont ouvert la voie, et bénéficiant des ressources de l'informatique, cette étude devrait s'appuyer sur la liste dressée par office, des officiers du roi et des seigneurs - liste

(8). - L'Histoire du sentiment national vient d'être renouvelée par la thèse de Ph. CONTAMINE, Guerre, Etat et Société à la fin du Moyen-Age, Paris, 1972. Le même auteur a publié dans la collection Que sais-je? une brillante synthèse La Guerre de Cent ans. R. JOUET, dans un ouvrage publié à Caen en 1969, a mis en évidence La Résistance à l'occupation anglaise en basse Normandie entre 1418-1450.

Etude de la création d'un mythe à travers le sentiment national: c'est ce que démontre l'ouvrage de G. DUBY, Le dimande Bouvines paru en 1973 à Paris dans la collection Les Trente jours qui ont fait la France.

Histoire aussi du personnel politique. Avec le recours aux méthodes informatiques, Fr. AUTRAND prépare sa thèse Les gens du Parlement de Paris à la fin du Moyen-Age. 1345-1454. 
grâce à laquelle seraient reconstituées les carrières, reconstruits les lignages, dépistée la mobilité géographique des individus et des familles, édifié, en définitive, un véritable fichier social à l'échelle de la France médiévale. Cette entreprise s'insérerait tout naturellement dans une plus vaste enquête sur les structures sociales et les pouvoirs politiques que les historiens des institutions ont envisagé d'entreprendre et dont l'objet final serait de rechercher quels groupes exercent effectivement le pouvoir dans les différents types de sociétés et de formes politiques .

De même que l'histoire proprement politique, l'histoire militaire connaît depuis plusieurs années un net regain de vitalité, non seulement parce que les chercheurs en ont désormais une conception plus large, mais aussi parce qu'ils pensent pouvoir sans trop mauvaise conscience, aborder un sujet longtemps considéré comme presque interdit. Transformée dans son esprit, l'histoire des guerres est devenue "polémologie". Cette renaissance affecte toutes les périodes et notamment le Moyen Age. A ce nouvel essor contribuent toutes les écoles historiques, selon des styles naturellement divers: Belgique, Suisse, Angleterre, Etats Unis, les écoles italienne et allemande restant légèrement en retrait. Dans ce domaine, et en dépit de la relative rareté des spécialistes, la France a connu récemment des réussites, qu'il conviendrait de consolider. La question est notamment ouverte de savoir quelles furent les répercussions de la guerre - réalité politique quasi permanente - sur les institutions de l'Etat médiéval et sur sa vie même. Si de remarquables recherches nous ont appris ce qu'avaient été les armées du roi de France au bas Moyen Age, elles ont également montré que la mise sur pied de ces armées n'avait représenté qu'une infime partie de "l'effort de guerre": celui-ci, défensif, reste avant tout d'initiative locale. Les autorités locales ont levé des troupes, elles ont surtout construit ou reconstruit des remparts. Dresser la carte des fortifications françaises au Moyen Age, dater leurs constructions, inventorier ce qu'il en reste, étudier leurs techniques, établir leur coût, les conséquences juridiques, financières et politiques de leur édification, tel pourrait être, dans le domaine considéré, un exemple de recherche. Mais il serait insuffisant de s'intéresser seulement à la guerre organisée et institutionnalisée de l'Etat triomphant: la guerre féodale a été guerre privée, vendetta; son étude mérite d'être reprise avec un regard neuf. 
Les querelles épiques entre sociologues et historiens qui ont longtemps marqué l'évolution des sciences humaines en France sont, on le constate, dépassées et oubliées. En tout domaine, l'historien tient aujourd'hui, non seulement des apports, mais de l'esprit même de la sociologie. L'histoire religieuse (9) est spécialement marquée par de telles orientations. Un effort est fait pour mieux comprendre le mécanisme des institutions ecclésiastiques, la portée réelle des réformes, la composition de la hiérarchie, grâce à l'identification des personnages, à la recherche de leurs attaches familiales, à la reconstitution de leurs carrières. Des préoccupations très contemporaines orientent les médiévistes français vers la quête et l'interprétation des manifestations de la piété: dévotions, dons, legs, images, pélerinages. On s'attache à découvrir ce qu'était la vie religieuse dans les couches les moins cultivées de la population. Non sans difficulté, car les sources sont rares, d'interprétation délicate, alors qu'il faut pourtant distinguer les niveaux culturels, pénétrer les croyances et interpréter les comportements religieux de populations illétrées et "muettes". Une grande attention est apportée aux hérésies, spécialement à celles du XIIIe siècle: tout un courant de pensée, qui dépasse largement les milieux universitaires et érudits, incite du reste à la sympathie pour les marginaux, les "contestataires", les persécutés qui attirèrent alors les croisés de la France du nord vers l' "Occitanie". L'enquête sur la pauvreté, lancée à la Sorbonne, et à laquelle ont participé tant d'historiens de toute nationalité, a fait large place aux pratiques religieuses. L'hagiographie - entendue comme moyen de repérage des idéaux d'une époque ou d'une catégorie sociale - laisse entrevoir des approches nouvelles de la spiritualité.

L'Eglise du haut Moyen Age, qui ne semblait plus susciter la curiosité, est retrouvée à travers l'un de ses représentants les plus remarquables, l'archevêque de Reims Hincmar. Des recherches d'ecclésiologie sont également en cours. Les tendances à l'érémitisme aux XIe-XIIe siècles provoquent encore des travaux. La diffusion des ordres mendiants, envisagée sous les angles géographique et chronologique, devrait encourager à mieux connaître Carmes et Ermites de

(9). - L'historiographie religieuse recouvre des aspects divers. La prosopographie est à l'honneur avec la thèse de J. DEVISSE: Hincmar, archevêque de Reims 845-882 qui sera publiée au cours du premier trimestre 1975 chez Droz à Genève. La thèse de $\mathrm{J}$. LONGERE, La prédication des maitres parisiens durant la seconde moitié du XIIe siècle, présente un grand nombre de sermons inédits. Elle sera publiée en 1975 aux Etudes augustiniennes. Publication de documents encore avec l'édition du Manuel des Inquisiteurs par $\mathbf{N}$. EYMERICH et F. PENA, Paris - La Haye 1973. L'édition des statuts synodaux se poursuit avec l'ouvrage d'O. PONTAL Les Statuts Synodaux français du XIIIe - Tome I: Statuts de Paris et Synodal de l'Ouest, Paris, 1971. 
Saint Augustin, tous délaissés au profit des Mineurs et des Prêcheurs. On s'est cependant intéressé aux Sachets et aux Spirituels dans l'ordre de Saint François. On commence à s'interroger sur le monachisme féminin. Aux XIVe et XVe siècles, les médiévistes tendent la main aux modernistes, pour apprécier la marche de la révolution qui a abouti à la Réformation et à la Réforme catholique.

Des publications récentes et le recours à l'informatique (10) devraient donner plus de vigueur à l'étude des conciles et des statuts synodaux, à défaut de la conservation des registres de visites pastorales dont la France est malheureusement beaucoup moins riche que l'Angleterre. L'application de techniques proches de celles qui sont employées pour l'occupation du sol permet de reconstituer la formation du réseau des paroisses rurales. Mais l'étude de la vie paroissiale n'est pas assez avancée: elle n'est pourtant pas impossible pour la fin du Moyen Âge, comme l'ont prouvé quelques démonstrations brillantes. On attend aussi une présentation globale de la vie religieuse à l'intérieur d'un diocèse ou d'une région, dans des limites chronologiques bien choisies.

Dans un domaine tout proche de l'histoire de l'Eglise, l'histoire de l'éducation, au sens le plus large continue d'être généralement négligée par les médiévistes français. La nature même des sources a

(10). - La recherche de rouvelles méthodes par l'utilisation de l'informatique a donné naissance à une collaboration franco-belge entre le CETEDOC de Louvain et la Sorbonne. Sous la direction de M. MOLLAT et de P. TOMBEUR sont publiés deux ouvrages: de LATRAN I à LATRAN IV, LYON I et LYON II, Concordances, Indiccs, Listes de fréquence, Paris - Louvain 1974. Un travail de synthèse de F. RAPP présente l'Eglise et la vie religieuse en Occident à la fin du Moyen-Age, Paris, 1971, dans la collection Nouvelle Clio. La liturgie et la pastorale semblent être deux sujets demandés. C. VOGEL traite Le pêcheur et la pénitence au Moyen-Age, Paris, 1969 et M. H. VICAIRE donne un aperçu de La pastorale des moeurs dans les conciles languedociens, publiés dans Les Cahiers de FANJEAUX, 1971. Y. DOSSAT s'est appliqué à définir L'hérésie en Champagne aux XIIe et XIIIe, Paris, 1969.

Enfin un ouvrage représentant dix années de recherche regroupe les études poursuivies au séminaire de M. MOLLAT: Recherches sur les pauvres et la pauvreté, Paris, 1974. Dans la collection des publications de la Sorbonne; il est composé de deux tomes, l'un s'attachant à définir la pauvreté spirituelle du monde mérovingien au XVe siècle, l'autre donnant des exemples des réalisations caritatives du XIIe au XVIe. Il faut noter en particulier le châpitre consacré par $C h$. M. de la RONCIERE à l'étude de Florence. Il inaugure une nouvelle méthode consistant à calculer le salaire des ouvriers en fonction du prix du pain et en nombre de calories. Tableaux d'autant plus parlants, qu'ils débouchent sur des réalités quotidiennes et permanentes. Enfin les rapports entre l'Eglise et la Société sont illustrés par l'ouvrage d'E. MAGNONNORTIER, La société laïque et l'église dans la province ecclésiastique de Narbonne de la fin du VIIIle à la fin du Xle siècle, publié à Toulouse, 1974. 
amené les chercheurs à s'orienter vers l'étude, plus aisée, de courants intellectuels, ou vers celle des institutions universitaires - laquelle se trouve d'ailleurs gênée, dans bien des cas, par le très mauvais état de la documentation.

L'étude des courants intellectuels (11) rejoint l'histoire de la philosophie, de la théologie et du droit et des sciences. La formation proprement littéraire des étudiants est très mal connue. L'histoire des Universités n'a guère fait de progrès en France (en contraste avec ce qui se passe à l'étranger), sauf deux ou trois exceptions - dont la plus importante est due à l'Université américaine de Notre Dame depuis la fin du XIXe siècle. La première tâche, une fois publiée la bibliographie de l'histoire des Universités françaises, sera d'établir un inventaire critique des sources, et de formuler un plan de publication. On essaie en ce moment de dresser un répertoire et une bibliographie des petites écoles, sur lesquelles on ne sait pratiquement rien. L'ignorance où nous sommes de l'organisation et du contenu de l'instruction élémentaire est une lacune grave: il est impossible aujourd'hui de savoir, en particulier, comment on apprenait le français avant le XVe siècle. Une prospection très large des sources archivistiques, littéraires, doctrinales relative à la formation de l'individu du Moyen Age est indispensable à toute l'histoire des mentalités .

Voilà lâché les maîtres mots de l'historiographie médiévale telle du moins qu'on la conçoit en France: "l'histoire des mentalités...".

Depuis le début du siècle, l'histoire a assimilé les apports successifs de la science économique et de la sociologie. Aujourd'hui, l'historien jette sur le passé le regard de l'ethnologue, et l'ethnologie historique "conduit naturellement à l'étude des mentalités, considérées comme ce qui change dans l'évolution historique".

L'histoire des mentalités (12) institue ainsi d'emblée une distinction entre son domaine historique propre, qui est celui de la vie quo-

(11). - Enfin deux ouvrages fondamentaux permettent la réflexion sur les problèmes de l'enseignement et de la vie universitaire: J. PAUL, Histoire intellectuelle de l'Occident médiéval, Paris, 1973 et J. VERGER, Les Universités au Moyen-Age, Paris, 1973.

(12). - On ne peut aborder l'histoire des mentalités, sans signaler d'abord le châpitre écrit par J. LE GOFF: Les mentalités, une histoire ambigüe, dans le tome III Nouveaux objets de l'ouvrage collectif publié sous la direction de J. LE GOFF et P. NORA Faire de l'histoire, Paris, 1974. Parmi 
tidienne, de la routine, des systèmes de valeur à la longue survie - et celui de l'histoire de la culture, qui est création et changement. Elle pose en principe que toute société connaît plusieurs milieux culturels, simultanés, qui se juxtaposent, s'interpénètrent, véhiculés par la langue, l'éducation, les mythes. Elle s'interroge par conséquent sur la nature des sources qu'elle peut utiliser et n'en découvre pas qui lui soient spécifiques. Toutes lui sont utiles: les chartes et les notices révélatrices non seulement par leur contenu, mais par leur aspect formel; les sources narratives, un moment négligées; la "littérature de divertissement"; l'objet archéologique - oeuvre d'art ou humble outil de la civilisation matérielle, l'emblème, tous révélateurs d'une certaine représentation du monde.

Les médiévistes français sont particulièrement sensibles à ce courant de recherche, dont les inspirateurs se réclament de leur discipline: l'intérêt pour le "mental collectif" anime et stimule de plus en plus leurs recherches. L'histoire des mentalités apparait en définitive comme une histoire totale et une explication ultime, visant à incorporer les observations des historiens de l'art et de la littérature, dépassant l'économique et le social, tirant parti de toutes les catégories de sources.

Dans cet aperçu trop sommaire, injuste à force d'être simplifié, j'ai bien conscience de passer sous silence des disciplines considérables. Est-il aujourd'hui concevable - quand on célèbre de tous côtés les mérites de l'interdisciplinarité - d'ignorer l'apport des historiens de la philosophie, des sciences, des littératures médiévales? Celui des archéologues... Est-il possible de donner le sentiment que l'histoire du Moyen Âge est seulement celle de l'Occident, alors qu'une brillante école de byzantinistes, riche d'hommes et de tradition existe en France? Il eût fallu pour traiter de l'ensemble de ces recherches à la fois plus de compétence et davantage d'espace.

Il importe toutefois d'évoquer, au terme de ce survol, ce qui est, à mes yeux, l'un des aspects les plus encourageants de la recherche actuelle en histoire médiévale: la renaissance des études érudites.

les études relevant de l'histoire des mentalités, citons J. HEERS, Fêtes, Jeux et Joutes dans les sociétés d'Occident à la fin du Moyen-Age, Paris, 1971. Ph. CONTAMINE, Contribution à l'histoire d'un mythe: les 170.000 clochers du royaume de France dans les Mélanges offerts à E. PERROY, Paris, 1973, ainsi que du même auteur: L'oriflamme de Saint-Denis aux XIVe et XVe; étude de Symbolique religieuse et royale dans les Annales, Paris, 1973. 
En effet, sous l'empire conjugué des novations techniques et méthodologiques, les sciences auxiliaires de l'histoire subissent en ce moment même un profond renouvellement.

Le regard nouveau jeté sur l'histoire fait reprendre vie à certaines d'entre elles, qu'on croyait discréditées. Telle l'héraldique, immense répertoire de symboles, qui commença d'être méprisée des historiens au moment même où l'école ethnologique française s'interessait aux "totems" des "primitifs". L'histoire des mentalités lui rend un sens historique et est à l'origine de son renouvellement, annoncé par des travaux rigoureux et très originaux. L'épigraphie médiévale, longtemps négligée en France, commence à y prendre corps et procède en ce moment au rassemblement de son matériel documentaire.

Des sciences, que l'on pouvait croire relativement figées, apparaissent soudain en pleine mutation. Il suffira d'évoquer, à titre d'exemple, la paléographie latine. Celle-ci se trouve actuellement en pleine évolution - pour ne pas dire révolution - dans ses buts comme dans ses méthodes. Depuis quelques temps déjà, de descriptive elle était devenue analytique; autrefois orientée vers la connaissance des formes et l'aspect extérieur des lettres, elle se préoccupe désormais de connaître la genèse et le sens de ces formes et les raisons de leurs modifications; l'attention se porte de plus en plus sur les menus détails - ponctuation, signes d'abréviations, cédilles, ligatures etc. - qui apparaissent comme significatifs. Un nouvel élément entre aujourd'hui en jeu: le développement rapide de la recherche codicologique, qui exerce une influence incontestable sur les paléographes. Dans certains domaines se rapportant à la constitution du manuscrit, la frontière entre codicologie et paléographie est floue, les points communs nombreux; les spécialistes portent leur regard audelà de l'écriture, produit fini sur une page, pour découvrir le processus de création, le temps d'écriture, les conditions de travail des copistes et leur organisation. Ils tendent à faire de leur "art" une science où interviennent des techniques de laboratoire.

Sans doute, l'expérience, le jugement et l'intuition des grands spécialistes demeurent-ils irremplaçables; mais eux-mêmes attachent le plus grand intérêt aux recherches de points de comparaison précis dans le temps comme dans l'espace - écritures datées et localisées et à tous les moyens permettant de remplacer les conclusions "ex cathedra" par de véritables expertises.

La photographie, pratiquée de longue date, est préférée de plus en plus au dessin. Utilisée à l'échelle 1 , elle permet les meilleures comparaisons d'écriture; et la macrophotographie, sous toutes ses for- 
mes, aide à analyser le "ductus", le geste exact du copiste, ses reprises de plume. L'emploi de la lampe de Wood pour l'examen direct de textes effacés s'est amélioré grâce aux appareils combinant une loupe éclairante avec un tube à rayons uv. La photographie sous lumière monochromatique, la radiographie et plus récemment encore la bétagraphie sont de pratique fréquente maintenant sinon courante.

D'autres méthodes plus rares, à leurs tous débuts, transformeront peut-être l'avenir, telles que l'identification des écritures à l'aide des hologrammes et du rayon laser; les calculs combinés de l'angle d'écriture, de la position de la plume, du rapport du copiste à son plan de travail.

Malgré de vives réticences chez beaucoup de paléographes, on en vient à admettre désormais l'emploi de l'informatique pour exploiter la masse des renseignements obtenus grâce à de multiples et complexes recherches de détails.

C'est dans cette perspective que les chercheurs ont pris conscience récemment de l'urgente nécessité de normaliser leur vocabulaire et ont formé le projet d'un lexique polyglotte.

En paléographie, en effet, la tendance est au regroupement des efforts et à la constitution de groupes de travail internationaux. Le même mouvement anime la diplomatique, dont les spécialistes viennent de créer une Commission internationale qui a déjà suscité plusieurs congrès et lancé des projets de publications communes.

"Commission internationale", "recherche commune": il serait outre cuidant, en effet, de vouloir considérer comme spécifiquement - voire même principalement française, la renaissance contemporaine de l'érudition. S'il est un domaine où s'exerce la solidarité, c'est bien celui des "sciences auxiliaires" de l'histoire. La tradition est ancienne: elle remonte à la singulière communauté que formèrent spontanément les érudits d'Occident dès la fin du XVIe siècle. L'échange est du reste plus que partout ailleurs nécessaire dans des études qui sont par la force des choses le patrimoine commun des médiévistes, au delà de toute frontière et de toute habitude intellectuelle nationale. Ainsi l'historiographie française dont je me suis appliqué à souligner les particularités, sinon les particularismes, se réinsère-t-elle dans un ensemble plus vaste. En France comme ailleurs, il apparaît bien que 
l'évolution des études médiévales va de plus en plus dépendre d'une méthodologie qui est, elle-même, en voie de profonde mutation. I est évident aussi qu'en France, comme ailleurs, aucune des transformations en cours ne pourra être pleinement réalisé et donner lieu à des oeuvres importantes, si n'est entendu l'appel universel des historiens pour la mise au jour, la publication et l'exploitation de nouvelles sources documentaires, narratives et archéologiques. Mais ceci est une autre histoire. Une histoire d'argent .

\section{CURRICULUM VITAE.}

JEAN GLENISSON. Né en 25 janvier 1921, à JONZAC (Charente-Maritime).

- Ancien. Professeur à la Faculdade de Filosofia de l'Université de São Paulo. 1958 et 1959.

- Directeur d'études à l'Ecole Pratique des Hautes Etudes (VIe section).

- Directeur de l'Institut de Recherche et d'Histoire des Textes.

\section{TRAVAUX.}

I. - OUVRAGES.

1) - Inicią̧ão aos Estudos Históricos. São Paulo, Difusão Européia do Livro, 1961, 350 p.

2 ) - 1300-1500. Le temps des périls. Les découvertes du monde, Paris, CAL, 1966. (Les métamorphoses de l'Humanité).

3) - Textes et documents d'Histoire du Moyen Age XIV e$X V e$ siècles. I. Perspectives d'ensemble. Les "crises et leur cadre". Paris, Sedes, 1970.

PUBLICATIONS DE DOCUMENTS.

a) - Clément VI (1342-1352). Lettres closes, patentes et curiales se rapportant à la France, Paris, de Boccard, 1958-1961. (Bibliothèque des Ecoles françaises d'Athenes et de Rome). [En collaboration avec G. MOLLAT et E. DEPREZ]. 
b) - Registres du Trésor des Chartes, Tome I. Règne de Philippe le Bel. Paris. Imprimerie Nationale, 1958 (Archives Nationales. Inventaires et documents). [sous la direction de $R$. FAWTIER, en collaboration avec J. GUEROUT].

c) - Correspondance administrative des légats et vicairesgénéraux dans les terres italiennes de l'Eglise. Tome ler. Albornoz et Androin de la Roche (1353-1367). (Bibliothèque des Ecoles françaises de Rome et d'Athenes). 